\title{
XXIII.
}

\section{Über das Vorkommen von Typhusbazillen im Liquor cerebrospinalis bei Typhuskranken.}

(Aus der Direktorialabteilung (Prof. B r a u e r) und dem Pathologischen Institut (Prof. Eug. Fra enkel) des Allgemeinen Krankenhanses Hamburg-Eppendorf.)

Von

Dr. Berthold Hannes.

Assistent am Patholog. Institut vom 1. November 1909 bis 1. April 1911, an der Direktorialabteilung vom Mai 1911 bis Oktober 1913.

Daß der Typhusbazillus imstande ist, unter uns noch nicht genügend bekannten Bedingungen eine echte Meningitis zu erzeugen, ist eine sowohl durch die klinische Beobachtung als auch durch den Sektionsbefund erhärtete Tatsache.

Seit der ersten Mitteilung von $Q u$ in $\mathrm{cke}$ und $\mathrm{St}$ üh $\mathrm{ler} \mathrm{r}^{1}$ über dieses Vorkommnis sind in der Literatur eine ganze Reihe von Fällen beschrieben worden, bei denen es entweder vital mittels der Lumbalpunktion und der bakteriologischen Beforschung der gewonnenen Zerebrospinalflüssigkeit oder postmortal durch die bakteriologische Verarbeitung des Exsudates der Hirnhäute gelungen ist, den Typhusbazillus als den Erreger dieser Meningitis typhosa nachzuweisen.

Von dieser durch den Typhusbazillus hervorgerufenen echten Meningitis soll hier nicht die Rede sein, sondern von dem Vorkommen von Typhusbazillen in einem sowohl makroskopisch als auch mikroskopisch normalen Liquor cerebrospinalis bei an Typhus abdominalis erkrankten Individuen.

Diese Untersuchungen bilden eine Ergänzung der Untersuchungen von Eug. Fra en kel${ }^{2}$ über das. Verhalten des Gehirns bei akuten Infektionskrankheiten. Fra e $\mathbf{n k e l}$ konnte bei der kulturellen Verarbeitung von sechs Gehirnen von an Typhus abdominalis verstorbenen Individuen in einem Falle Typhusbazillen nachweisen, während die fünf übrigen Gehirne steril waren.

Die bakteriologische Untersuchung der Gehirne von an Typhus verstorbenen Patienten sind nach der von Fra e $\mathrm{kel}$ angegebenen Methode im HamburgEppendorfer Pathologischen Institut fortgesetzt. In den Jahren 1910, 1912 und 1913 wurden unter 41 bakteriologisch untersuchten Gehirnen 9mal Typhusbazillen gefunden.

Die Literatur über den Nachweis von Typhusbazillen im Liquor ohne sonstige Veränderungen in diesem ist nicht sehr reichhaltig, dafür finden sich aber so unkritische und einander widersprechende Angaben niedergelegt, $d a b$ auf dieselbe kurz eingegegangen werden muß.

Um es gleich vorwegzunehmen, sei betont, daß von einem Fall, der zu der in Rede stehenden Gruppe gerechnet werden soll, verlangt werden muß, daß in 
dem klaren und auch nicht mit den geringsten Spuren von Blut verunreinigten Liquor in einwandsfreier Weise durch die üblichen bakteriologischen Untersuchungsmethoden die Anwesenheit des Typhusbazillus nachgewiesen sein muß. Die makroskopische und mikroskopische Abwesenheit von Elementen des Körperblutes muß unseres Erachtens unbedingt gefordert werden, denn bei der fast regelmäBigen Anwesenheit der Typhusbazillen im Blut der Typhuskranken ist der Einwand, daß die gefundenen Bazillen erst sekundär bei der Entnahme aus dem Blut hineingekommen sind, nicht zu widerlegen. Wie verhalten sich nun diesen Forderungen gegenüber die in der Literatur gemachten Angaben?

Was die Fälle mit negativen Resultaten anbelangt, so teilen $\mathrm{Wilms}^{3}$ einen Fall, Salomon ${ }^{4}$ vier Fälle und $\mathrm{Sch} 0$ enborn ${ }^{5}$ zwei Fälle mit, wo bei klarem Liquor die bakteriologisehe Untersuchung auf Typhusbazillen erfolglos blieb. Die klinischen Erscheinungen waren Benommenheit, Nackenstarre, Schwindel, Erbrechen und heftige Kopfschmerzen. Auf eine Kritik der bakteriologischen Technik soll nicht eingegangen werden.

Die mir bekanntgewordenen positiven Fälle sind die folgenden:

L e w k ow i z , 7jähriges Kind mit Nackenstarre und Benommenheit, Ausgang in Heilung; im Blut Typhusbazillen, in dem mit Blat verunreinigten Liquor ebenfalls Typhusbazillen Der Fall kann also nicht als einwandsfrei betrachtet werden. S e h ü t z $\mathrm{e}^{7}$ beschreibt zwei Fälle von dem mandschurischen Kriegsschauplatz mit Benommenheit, Nackensteifigkeit und klarem Liquor, in dem durch die Kultur Typhusbazillen nachgewiesen wurden, und zwar zu einer Zeit, wo die bakteriologische Untersuchung des Blutes und des Stuhles noch keine positiven Resultate ergeben hatte. S c hü $t \mathrm{z}$ e empfiehlt aus diesem Grunde die Lumbalpunktion zu diagnostischen Zwecken. Beide Patienten kamen zur Heilung. S i l b e r b e $\mathrm{r}^{8}{ }^{8}$ konnte unter neun Fällen mit Benommenheit und klarem Liquor in einem Falle kulturell und in sieben. Fällen mikroskopisch Typhusbazillen nachweisen; es ișt selbstverständlich, daß̣ die in den sieben Fällen im Zentrifugat nachgewiesenen beweglichen Stäbchen nicht mit genügender Sicherheit als Typhusbazillen erwiesen sind, andererseits ist es sehr unwahrscheinlich, dabie sieh in allen Fällen, wo sie mikroskopisch sichtbar waren, dem kulturellen Nachweis mittels Anreicherung in Gallenpepton entzogen haben sollten. Bei dem Fall von $\mathrm{N}$ i e t e $\mathrm{r}^{9}$ handelt es sich um post mortem entnommene Zerebrospinalflüssigkeit mit Beimengungen von Blut. Der Patient war unter meningitischen Erscheinungen zugrimde gegangen; da keine Sektion vorliegt, läßt sich über die Beschaffenheit der Hirnhäute nichts aussagen und also weder eine echte Meningitis noch eine zufällige Infektion durch das Blut ausschlieBen.

Das von mir untersuchte Material umfaßt 42 Fälle. 11 Fälle verdanke ich der Liebenswürdigkeit des Herrn Marinestabsarzt Dr. Kitt m a n $\mathfrak{n}$, der vor mir die Typhusabteilung inne hatte, die übrigen Fälle wurden von mir selbst behandelt und die Lumbalpunktion entweder von mir selbst oder unter meiner Leitung vorgenommen. In einem Fall, III, 5, wurde zweimal punktiert.

Die kulturelle Untersuchung wurde in der Weise vorgenommen, daß $1-3 \mathrm{ccm}$ Liquor in zwèi Röhrchen mit Rindergalle gegeben und aus diesen Röhrchen nach 24, 48 und 72 Stunden Ausstriche auf den C o n r a d i - D r ig a ls k i - Nährboden gemacht wurden. Die Identifizierung der gewachsenen Bakterien geschah in der üblichen Weise auf Traubenzuckeragar, Neutralrotagar, Kartoffel, Lakmusmolke, Peptonwasser und Milch. 
AuBerdem wurde noch in 28 Fällen der Druck des Liquor gemessen und in 22 Fällen der Druck gemessen, die Zählung der Zellen in der F u c h s - R o s.e n thal schen Kammer und die Nonne-A p peltsche Globulinreaktion vorgenommen.

Die bakteriologische Untersuchung des Blutes und die Agglutinationsreaktion wurde bei sämtlichen Fällen angestellt.

Bei den zur Sektion gekommenen Fällen wurde mit Ausnahme von zwei Fällen, bei denen die Sektion des Schädels nicht gestattet war, die bakteriologische Cntersuchung des Gehirns vorgenommen.

Es erschien für die vorliegende Untersuchung zweckmäBig, die Fälle, die jetzt kurz mit den notwendigsten Daten mitgeteilt werden sollen, nach dem Verhalten des Sensorium mitzuteilen; es wurden deshalb drei Gruppen unterschieden:

1. Fälle mit völlig freiem Bewußtsein,

2. Fälle mit leichter Benommenheit,

3. Fälle mit vollkommener Benommenheit.

Die einzelnen Fälle verteilen sich auf die verschiedenen Gruppen folgendermaßen: 24 Fälle auf Gruppe I, 8 auf Gruppe II und 10 auf Gruppe III.

\section{Fälle der Gruppe I.}

1. H., 32 J., ô. Leichte Kopfschmerzen. Blut nach $2 \times 24$ Stunden steril, Lumbalflüssigkeit nach $3 \times 24$ Stunden steril.

2. L., 16 J., §. ' Im Blut Bacterium typhi, Lumbalflüssigkeit nach $3 \times 24$ Stunden steril.

3. Gr., $23 \mathrm{~J}$. , of. Rückenschmerzen. Im Blnt Bacterium typhi. In $\mathrm{d} \in \mathrm{r} \mathrm{L}$ u mbal fl üssigkeit Bacterium typhi, bei der Lumbalpunktion kam etwas Blut in die Lumbalflüssigkeit.

4. M., 27 J., ơ. Leichte Kopfschmerzen. Im Blut Bacterium typhi. Lumbalfiüssigkeit nach $3 \times 24$ Stunden steril.

5. N., 27 J., ô. Im Blut Bacterium typhi, Lumbalflüssigkeit nach $3 \times 24$ Stunden steril.

6. V., 28 J., o. Im Blut Bacterium typhi, Lumbalflüssigkeit nach $3 \times 24$ Stunden steril.

7. D., 25 J., đิ. Im Blut Bacterium typhi. Lumbalflüssigkeit nach $3 \times 24$ Stunden steril.

8. B., $25 \mathrm{~J}$., of. Blut steril, Lumbalflüssigkeit nach $3 \times 24$ Stunden steril.

9. P., 29 J., ô. Blut am 13. Krankheitstage Bacterium typhi, am 15. steril. Lumbalfiüssigkeit am 15. Tage bei $40^{\circ} \mathrm{C}$ nach $3 \times 24$ Strunden steril.

10. L., $18 \mathrm{~J}$, $\delta^{*}$ : Blut am 15. Krankheitstage steril. Lumbalflüssigkeit am 15. Krankheitstage bei $40,2^{\circ} \mathrm{C}$ steril, Druck 110, Zellgehalt 2, Nonne-Appelt negativ.

11. Sch., 24 J., ô. Blut am 8. Krankheitstage steril, Lumbalflüssigkeit am 9. Krankheitstage bei $39^{\circ} \mathrm{C}$ steril, klar, Druck 100 .

12. L., 20 J., $\hat{\text { o. }}$ Blut am 8. Krankheitstage Bakterium typhi. Lumbalfüssigkeit am 9. Krankheitstage bei $40,2^{\circ} \mathrm{C}$ nach $3 \times 24$ Stunden steril, klar, Druck 130, Zellgehalt 2, NonneAppelt negativ.

13. Sch., 27 J., $\hat{0}$. Geringe Kopfschmerzen, im Blut am 7. Krankheitstage Bacterium typhi, Lumbalflüssigkeit bei $39,8^{\circ} \mathrm{C}$ am 7 . Krankheitstage steril, klar, Druck 200, Zellgehalt 1, Nonne-Appelt negativ.

14. R., 22 J., of. Geringe Kopfschmerzen, im Blut am 9. Krankheitstage Bacterium typhi, Lumbalflüssigkeit bei $40^{\circ}$ C. am 10. Krankheitstage steril, klar, Druck 160, Zellgehalt 5 , Nonne-Appelt negativ. 
15. Seh., 30 J., $\hat{o}$. Im Blut am 5. Krankheitstage Bacterium typhi, Lumbalflüssigkeit am 8. Krankheitstage bei $40,2^{\circ} \mathrm{C}$ steril, klar, Druck 170 , Zellgehalt 2, Nonne-Appelt negativ. Tod am 36. Krankheitstage, Sektion verweigert.

16. K., 48 J., ơ. Im Blut am 12. Krankheitstage Bacterium typhi, Lumbalflüssigkeit am 14. Krankheitstage bei $40,2^{\circ} \mathrm{C}$ steril, klar, Druck 90 , Zellgehalt 10 , Nonne-Appelt negativ.

17. S., 18 J., $\delta$. Starke Kopischmerzen, im Blut am 9. Krankheitstage Bacterium typhi, Lumbalfüssigkeit am 11. Krankheitstage bei $40,2^{\circ} \mathrm{C}$ steril, klar, Druek 50.

18. M., 19 J., đ. Blut am 6. Krankheitstage, Lumbalflüssigkeit am 7. Krankheitstage bei $39,7^{\circ} \mathrm{C}$ steril, klar, Druck 50, Zellgehalt 11 , Nonne-Appelt negatir.

19. S., 10 J., đ. Im Blut am 4. Krankheitstage Bacterium typhi, Lumbalflüssigkeit am 5. Krankheitstage steril, klar, Druck 150, Zellgehalt"5, Nonne-Appelt negativ.

20. W., $20 \mathrm{~J}$., $\mathrm{O}^{\star}$. Blut am 14. Krankheitstage, Lumbalfiüssigkeit am 17. Krankheitstage bei $39,8^{\circ} \mathrm{C}$ steril, klar, Druck 105 , Zellgehalt 8 , Nonne-Appelt negativ.

21. E., 38 J., ơ. Im Blut am 14. Krankheitstage Bacterium typhi, Lumbalflüssigkeit am 21: Krankheitstage bei $38,6^{\circ} \mathrm{C}$ steril, klar, Druck 180, Zellgehalt 5, Nonne-Appelt negativ.

22. W., 15 J., o. Im Blut am 13. Krankheitstage Bacterium typhi, Lumbalflüssigkeit am 15. Krankheitstage bei $37,8^{\circ} \mathrm{O}$ steril, leicht blutig gefärbt, Druck 120 .

23. A., 28 J., 3. Im Blut am 8. Krankheitstage Bacterium typhi, Lumbalflüssigkeit bei $39,6^{\circ} \mathrm{C}$ am 10. Krankheitstage steril, klar, Druck 60, Zellgehalt 6, Nonne-Appelt negativ.

24. P., $18 \mathrm{~J}$., $\hat{0}$. Blut am 10., Lumbalflüssigkeit am 11. Krankheitstage bei $40,3^{\circ} \mathrm{C}$. steril, klar, Druck 80 , Zellgehalt 3, Nonne-Appelt negativ.

\section{Fälle der Gruppe II.}

1. Seh., 18 J., of. Im Blut Bacterium typhi, Lumbalfüssigkeit steril.

2. P., 27 J., $0^{x}$ Im Blut Bacterium typhi, Lumbalflüssigkeit steril. Tod am 9. Krankheitstage. Die Leichenuntersuchung ergibt im Blut Bacillus mucosus capsulatus und Diplococcus lanceolatus, in Milz und Brustwirbeln Bacterium typhi in Reinkultur.

3. W., 19 J., $\widehat{~}$. Im Blut am 14. Krankheitstage Bacterium typhi, Lumbalfüsssigkeit am 15. Krankheitstage bei $40,2^{\circ} \mathrm{C}$ steril, klar, Druck 50, Zellgehalt 1 , Nonne-Appelt negativ. Tod. am 19. Krankheitstage. Die Ieichenuntersuchung ergibt im Blut Bacillus phlegmones emphysematosae und Bacillus mueosus capsulatus, in der Galle Bacterium typhi in Reinkultur, in den Meningen Bacillus phlegmones emphysematosae, im Gehim Bacillus mucosus capsulatus, Staphylococcus aureus und Streptococcus haemolyticus.

4. R., $29 \mathrm{~J}$, హ. Blut am 8., Lumbalflüssigkeit am 9. Krankheitstage bei $39,2^{\circ} \mathrm{C}$ steril, klar, Druck 140, Zellgehalt 2, Nonne-Appelt negativ.

5. K., $15 \mathrm{~J} .$, o. Blut am 8., Lumbalflüssigkeit am 11. Krankheitstage bei $40,2^{\circ} \mathrm{C}$ steril, klar, Druek 130, Zellgehalt 9, Nonne-Appelt negativ.

6. Sch., 19 J., of. Blut am 9., Lumbalflüssigkeit am 10. Krankheitstage bei $39,5^{\circ} \mathrm{C}$ steril, klar, Druck 170, Zellgehalt 3, Nonne-Appelt negativ.

7. S., 20 J., ㅇ. Im Blut am 4. Krankheitstage Bacterium typhi, Lumbalfiüssigkeit am. 12. Krankheitstage bei $40,5^{\circ} \mathrm{C}$ steril, bei der Entnahme mit Blut verunreinigt.

8. H., 18 J., ठో. Im Blut am 3. Krankheitstage Bacterium typhi, Lumbalflüssigkeit am 5. Krankheitstage bei $40,2^{\circ} \mathrm{C}$ steril, klar, Druck 170, Zellgehalt 2, Nonne-Appelt negativ.

\section{Fälle der Gruppe III.}

1. C., $23 \mathrm{~J} .$, ơ. Im Blut und in der Lu mbalf 1 üs sigkeit Bacteriu $\mathrm{m}$ ty phi. Tod am 12. Krankheitstage. Leichenuntersuchung 18 Stunden na.eh dem Tode im Blut Bacterium typhi in Reinkultur, in Brustwirbel, Galle und Milz Bacterium typhi in Reinkultur, Gehirn steril.

2. C., $18 \mathrm{~J}$, of. Im Blut am 4. Krankheitstage Bacterium typhi, in der L u mbal flüssigkeit, die bei der Entnahme mit wenig Blut verunreinigt wurde, am 7. Krankheitstage bei $40,6^{\circ}$ Bacterium typhi. 
3. M., 28 J., ơ. Im Blut am 21. (?) Krankheitstage Bacterium typhi, in der L u m b a 1 fl î s sig k e i t am 23. Krankheitstage bei $40,2^{\circ} \mathrm{C}$ B a c teriu m ty phi, klar, Druck 100 , Zellgehalt 9, Nonne-Appelt negativ.

4. B., 55 J., $\hat{o}^{+}$Im Blut am 16. Krankheitstage Bacterium typhi, Lumbalflüssigkeit am 24. Krankheitstage bei $39,6^{\circ} \mathrm{C}$ steril, klar, Druck 130, Zellgehalt 2, Nonne-Appelt negativ. Tod am 59. Krankheitstage. Die Leichenuntersuchung ergibt im Blut Bacterium typhi, im Gehirn Bacillus mucosus capsulatus und Bacterium coli, in Milz, Galle und Nierenabsze B Bacterium typhi.

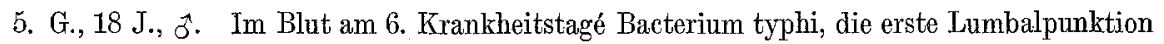
am 8. Krankheitstage bei $39,8^{\circ} \mathrm{C}$ und die zweite Lumbalpunktion am 20. Krankheitstage bei $40^{\circ} \mathrm{C}$ ergeben sterile Flüssigkeiten; die erste Flüssigkeit ist mit Blut verunreinigt, die zweite klar, Druck 120, Zellgehalt 5, Nonne-Appelt negativ. Tod am 34. Krankheitstage, Sektion nicht gestattet.

6. L., 19 J., 3. Im Blut am 10. Krankheitstage Bacterium typhi, die Lumbalpunktion am 11. Krankheitstage bei $40,2^{\circ} \mathrm{C}$ steril, klar, Druck 110. Tod am 19. Krankheitstage, Sektion nicht gestattet.

7. K., 32 J., б. Im Blut am 10. Krankheitstage Bacterium typhi, die Lumbalflüssigkeit am 11. Krankheitstage bei $40,2^{\circ} \mathrm{C}$ stexil, klar, Druck 70, Zellgehalt 9, Nonne-Appelt negativ. Tod am 22. Krankheitstage. Die Leichenuntersuchung ergibt im Blut, in der Milz, der Galle und der Niere Bacterium typhi, das Gehirn bleibt steril.

8. G., 16 J., ô. Im Blut am 10. Krankheitstage Bacterium typhi, die Lumbalflüssigkeit am 11. Krankheitstage bei 40,6 $\mathrm{C}$ steril, mit Blut verunreinigt, Druck 100.

9. H., 29 J., ठ․ Im Blut am 14. Krankheitstage Bacterium typhi, die Lumbalfüssigkeit am 16. Krankheitstage bei $40,3^{\circ} \mathrm{C}$ steril, klar, Druck 210 , Zellgehalt 5 , Nonne-Appelt negativ.

10. K., 19 J., J. Das Blut bei der ersten Entnahme am 5. Krankheitstage steril, am 8. Krankheitstage Bacterium typhi, die Lumbalfüssigkeit am 11. Krankheitstage steril, klar, Druck 100, Zellgehalt 2, Nonne-Appelt negativ. Tod am 13. Krankheitstage. Die Leichenuntersuchung ergibt in Blut und Galle Bacterium typhi in Reinkultur.

T a belle 1 .

\begin{tabular}{c|c|c|c|c}
\hline Gruppe & Zahl der Fälle & $\begin{array}{c}\text { Bact. typhi im } \\
\text { Liquor }\end{array}$ & $\begin{array}{c}\text { Bact. typhi im } \\
\text { Blut }\end{array}$ & Mortalität \\
\hline I & 24 & $(1): 4,17 \%$ & $17: 70,85 \%$ & $1: 4,17 \%$ \\
II & 8 & $-10: 50 \%$ & $2: 25 \%$ \\
III & 10 & $2(3): 20(30) \%$ & $10: 100 \%$ & $6: 60 \%$
\end{tabular}

Aus der Zusammenstellung und der Tabelle 1 ergibt sich, daß es unter den 42 untersuchten Fällen im ganzen viermal gelungen ist, im Liquor Typhusbazillen durch die Kultur nachzuweisen. Von diesen 4 Fällen müssen 2 Fälle ausgeschieden werden, da bei ihnen der Liquor bei der Punktion mit Blut verunreinigt wurde und in dem Blut dieser Fälle sich Bacterium typhi fand. Von den 4 positiven Fällen gehören 3 zur Gruppe III und ein Fall zur Gruppe I; nach Abzug der zwei nicht einwandsfreien Fälle verbleiben 2 Fälle ausschließlich für Gruppe III.

Die Punktionen wurden am 7., 12. und 23. Krankheitstage vorgenommen, bei dem 4. Fall kann der Krankheitstag nicht angegeben werden.

Im Blut wurde Bacterium typhi im ganzen $31 \mathrm{mal}==75,61 \%$, in den uns hier besonders interessierenden Fällen mit positivem Liquorbefund jedesmal gefunden. Der Zwischenraum zwischen der Blutentnahme und der Lumbalpunktion betrug in dem einen Fall 2, in dem anderen Fall 6 Tage. 
Die Gesamtmortalität betrug 21,43\%, die Mortalität in den einzelnen Gruppen $4,17,25$ und $60 \%$.

Von den beiden positiven Fällen kam Fall C, Gruppe III, 1, zum Exitus und zur Sektion. Bei der bakteriologischen Leichenuntersuchung wurden im Blut, Knochenmark, Galle und Milz Typhusbazillen in Reinkultur gefunden, während das Gehirn steril blieb. Die Lumbalpunktion wurde an demselben Tage, an dem der Exitus erfolgte, vorgenommen, die Sektion erfolgte 18 Stunden nach dem Exitus und trotzdem wurde das Gehirn frei von Typhusbazillen gefunden. Bei dem nahen Zusammenhang von Gehirn, Hirnhäuten und Liquor wäre ein positiver Befund im Gehirn zu erwarten gewesen. Es ist entweder möglich, daß die Bazillen überhaupt nicht ins Gehirn gelangt sind oder daß sie dort in einer so geringen Menge vorhanden waren, daß sie sich dem Nachweis mit der angewandten Methode entzogen; die Annahme, daß sie während des Lebens im Gehirn vorhanden waren und postmortal zugrunde gingen, hat nicht viel Wahrscheinlichkeit für sich. Es ergibt sich aus den negativen Befunden im Gehirn bei positiven Blutbefunden, daß von einem bloßen postmortalen Einschwemmen der Bakterien ins Gehirn nicht die Rede sein kann.

Es ist selbstverständlich nicht angängig, aus der geringen Zahl der positiven Liquorbefunde irgendwelche diagnostische und prognostische Schlüsse ziehen zu wollen.

Etwas anderes ist es mit der Frage des Zusammenhanges der bei den beiden Fällen ganz besonders schweren Bewußtseinstrübung und dem Vorhandensein der Typhusbazillen im Liquor. Ob es sich hierbei um rein toxische Einwirkungen oder um den psychischen Ausdruck der von Eug. Fraenkel bei der histologischen Untersuchung von Typhusgehirnen gefundenen entzündlichen Infiltrationen in den Hirnhäuten und in den Wandungen einzelner Hirnarterienäste handelt, läßt sich nicht entscheiden; auf jeden Fall wird man sich aber in Anbetracht der relativ hohen Záhl' der positiven Liquorbefunde bei den Fällen mit erheblicher Bewußtseinsstörung der Annahme zuneigen müssen, daß zwischen diesen beiden Tatsachen ein Zusammenhang besteht.

$\mathrm{T}$ abelle 2.

\begin{tabular}{c|c|c|c|c}
\hline \multirow{2}{*}{ Gruppe } & \multicolumn{2}{|c|}{ Druck } & \multicolumn{2}{c}{ Zellgehalt } \\
& $50-120$ & $121-210$ & $1-9$ & $10-11$ \\
\hline I & 9 & 5 & 10 & 2 \\
II & 1 & 4 & 4 & 0 \\
III & 6 & 2 & 6 & 0
\end{tabular}

Tab. 2 gibt eine Übersicht der Werte für den Druck und den Zellgehalt bei den einzelnen Gruppen. Aus den geringen Abweichungen nach oben lassen sich keine bindenden Schlüsse ziehen. Die Non n e - A p p e l tsche Reaktion wurde in allen untersuchten Fällen negativ gefunden.

Jedenfalls ergibt sich aus diesen morphologischen, physikalischen und chemischen Liquoruntersuchungen, daß in keinem unserer Fälle Veränderungen im Sinne einer Meningitis bestanden haben. 
$\mathrm{Zus}$ a m m enf as ung.

Unter 41 bakteriologisch untersuchten sonst normalen Zerebrospinalflüssigkeiten bei an Typhus abdominalis erkrankten Individuen konnten zweimal Typhusbazillen nachgewiesen werden.

Es besteht ein gewisser Parallelismus zwischen der Schwere der Bewußtseinstrübung und dem Vorkommen der Typhusbazillen in der Zerebrospinalflüssigkeit.

Die Lumbalpunktion hat für die Diagnose des Typhus abdominalis keine Bedeutung.

Prognostische Schlüsse können aus dem Nachweis von Typhusbazillen in der Zerebrospinalffüssigkeit nicht gezogen werden.

\section{Literatur.}

1. Qu i n cke-Stühler, Berl. klin. Wsehr. 1894, Bd. 15. - 2. Eu g. F r a enkel, Vireh. Arch. Bd. 194, Beiheft. -3. W il m s, Münch. med. Wschr. 1897, Bd. 3. -4. S a 1 o m o n, Berl. klin. Wschr. 1900, Bd.17. - 5. S c h o e n b or n, Med. Klin. 1906, Bd. 23 u. 24. -6. L e w $\mathrm{k} 0$ w i c z, Jahrb. f. Kinderheilk. 1902, Bd. 55. - 7. S c h ü t z e, Berl. klin. Wschr. 1905, Bd. 47. - 8. S i l b e r b er g, Berl. klin. Wschr. 1908, Bd. 29. - 9. N i e ter, Münch. med. Wschr. 1908, Bd. 19.

\section{XXIV. \\ Ein Beitrag zur Lehre vom Puerperalfieber und zur Be- handlung des fieberhaften Abortes.}

(Aus der Universitäts-Frauenklinik zu Jena.)

Von

Professor $\mathrm{M}$ a x He n kel.

„Kindbettfieber ist eine Spaltpilzerkrankung aller jener Wunden, die an den Geburtsorganen vom Damm bis in die Gebärmutterhöhle hinein entstehen." So definiert $\nabla$. Herff im W in ekel schen Handbuch den Begriff des Kindbettfiebers, und ich glaube kaum, daß es möglich ist, diesen präziser zu fassen.

Noch alle Jahre sterben in Deutschland zwischen 5- und 6000 Frauen an Kindbettfieber. Eine Besserung ist trotz unserer doch recht genauen Kenntnis der anatomischen und klinischen Tatsachen, um die es sich hierbei handelt, nicht eingetreten, und man muß sich fragen, woran das liegt. Wir kennen die Infektionserreger, kennen die Vorgänge; die sich bei der Infektion selbst abspielen, wissen sehr genau, wie sich die puerperale Infektion im Körper ausbreitet, könnten also Prophylaxe treiben, so weit gehend, daß schlechterdings das Puerperalfieber zu den überwundenen Erkrankungen gehören könnte; und doch ist das nicht so! Speziell in der letzten Zeit scheint es, als ob die Zahl der Puerperalfieberfälle wieder zunähme. Und zwar dürfte das damit zusammenhängen, daß prozentualiter eine 